\title{
Toll-like receptor 9 agonist stimulation enables osteogenic differentiation without altering the immune status of umbilical cord mesenchymal stem cells
}

\author{
YONGFENG YANG, YANWEN WANG, LI LI, JI BAO, FEI CHEN and LI ZHANG \\ Laboratory of Pathology, Department of Pathology, West China Hospital, \\ Sichuan University, Chengdu, Sichuan 610041, P.R. China
}

Received August 23, 2014; Accepted May 13, 2015

DOI: $10.3892 / \mathrm{mmr} .2015 .4429$

\begin{abstract}
Mesenchymal stem cells (MSCs) possess three characteristics critical to tissue regeneration; multipotency, low immunogenicity and an undifferentiated status, providing plasticity. However, increasing evidence has indicated that induction of an immune response in vivo can injure and reject MSC-based tissues. Toll-like receptors (TLRs) are a family of pathogen-associated pattern recognition receptors, which are important in bridging the innate and adaptive immune responses. TLRs have been demonstrated to exhibit important MSC biological regulatory functions in previous studies. To confirm the role of TLR9 in the immune status maintenance of MSCs isolated from umbilical cords, the present study performed assessments to detect agonist effects. Following addition of the TLR9 agonist, $\mathrm{CpG}$, to an umbilical cord mesenchymal stem cell (UCMSC) culture medium, a number of methods were used to detect the changes in the biological function of the UCMSCs. Reverse transcription-quantitative polymerase chain reaction indicated increased levels of pro-inflammatory molecules and decreased expression levels of stem cell markers following exposure to the TLR9 agonist. However, flow cytometry revealed that activation of TLR9 had no effect on the proliferation of peripheral blood leukocytes or the expression of surface markers. The present study also identified that $\mathrm{CpG}$-oligodeoxynucleotide promoted MSC osteogenic differentiation, while inhibiting MSC proliferation and migration. These results indicated that TLRs and their ligands may serve as regulators of MSC proliferation and differentiation, and affect the maintenance of MSC multipotency.
\end{abstract}

Correspondence to: Mr. Li Zhang, Laboratory of Pathology, Department of Pathology, West China Hospital, Sichuan University, 37 Guoxue Street, Chengdu, Sichuan 610041, P.R. China

E-mail: zhangli7375@scu.edu.cn

Key words: umbilical cord mesenchymal stem cells, Toll-like receptor $9, \mathrm{CpG}$, immunogenicity, cell differentiation

\section{Introduction}

Adult mesenchymal stem cells (MSCs) can be used as an innovative tool in cell-based therapy for degenerative disorders, chronic inflammatory and autoimmune diseases, and allograft rejection. MSCs have been isolated from several different tissues, including bone marrow, adipose, neuronal, amniotic, placental and Wharton's jelly of the umbilical cord (1). Self-regeneration and multi-lineage potential for differentiation are essential characteristics of MSCs (2). The low expression levels of co-stimulatory molecules, including CD80, CD86 and CD40, is another important feature of MSCs. The fourth important feature is that MSCs inhibit the activation, proliferation and function of immune cells, including T-cells, B-cells, natural killer-cells and antigen-presenting cells (3). These features render MSCSs an attractive cell-based therapeutic tool for developmental defects, degenerative diseases and mesodermal tissue injury, including bone, cartilage and muscle injury (4-11). However, the results of previous clinical trials investigating MSCs have not been encouraging, and one explanation for this is the recognition of MSCs in vivo by the host immune system, leading to injury and a marked reduction in activity $(12,13)$.

Toll-like receptors (TLRs) are a family of pathogen-associated pattern recognition receptors, which are important in the induction of effective immune responses. The conserved pathogen-derived components and endogenous ligands, also termed 'danger signals' activate TLRs (14). Distinct microbial products from bacteria, viruses and fungi are recognized by 11 TLR subfamilies in human cells. Among these, TLR9 recognizes CpG-DNA from bacteria and DNA viruses (15). MSCs express TLRs and elicit a number of biological functions in MSCs. Activation of TLR3 and TLR4 in MSCs isolated from adipose tissue increases osteogenic differentiation without impairing the immunogenic and immunosuppressive properties of MSCs (16). In addition, the migration of MSCs increases under activation by TLR8 and TLR9 (17).

At present, the sourcing of MSCs for clinical trials relies primarily on bone marrow, which is inherently limited by the invasive collection procedure for bone marrow MSCs (BMMSCs), and by their reduced self-renewal and proliferative abilities (18). Clinical trials have 
demonstrated the safe and beneficial application of UCMSC treatment in graft, vs. host-disease and systemic lupus erythematosus $(2,19,20)$. In the present study MSCs were isolated from umbilical tissue and stimulated with the TLR9 agonist, CpG-oligodeoxynucleotide (CpG-ODN), in order to determine whether activation of the TLR9 pathway affects the immune status and biological functions of UCMSCs. Co-culturing UCMSCs with peripheral blood leukocytes (PBLs) was used to measure the proliferation of PBLs following TLR9 activation. Flow cytometry was conducted to determine whether activation of the TLR9 pathway could increase the expression of CD80 and CD86. Antibody array and reverse transcription-quantitative polymerase chain reaction (RT-qPCR) were performed to assay the secretion of cytokines in the supernatant, and to determine the mRNA expression levels of immune-associated molecules in the presence of a TLR9 agonist.

\section{Materials and methods}

Isolation and culture of human UCMSCs. UCMSCs were purchased from the Sichuan Umbilical Cord Blood Stem Cell Bank (Chengdu, China). The cells were cultured in an incubator at $37^{\circ} \mathrm{C}$, with $5 \% \mathrm{CO}_{2}$ and saturated humid air, and the medium was replaced three times each week. Once the cell density exceeded 70\%, TrypLE Express (Gibco-BRL, Grand Island, NY, USA) was used to digest the cells, following which the cells were subcultured. The present study was approved by the ethics committee of West China Hospital, Sichuan University (Chengdu, China).

Immunofluorescence assay. The cells were seeded onto coverslips and fixed with neutral-buffered formalin for $15 \mathrm{~min}$. The cells were then permeabilized in $0.1 \%$ Triton X-100 for 10 min and incubated with monoclonal antibodies against stage-specific embryonic antigen 4 (SSEA-4; cat. no. Ab16287; Abcam Cambridge, UK). Immunofluorescence analysis was then performed and the immunostained cells were visualized using a Bio-Rad A1S1 laser confocal microscope (Bio-Rad Laboratories, Inc., Hercules, CA, USA).

Stimulation of MSCs using TLR9 agonists. The TLR9 reagent, CpG (Hycult Biotech, Inc., Plymouth Meeting, PA, USA), was dissolved in DMSO at a storage concentration of $25 \mathrm{mg} / \mathrm{ml}$. This was added to the UCMSC culture medium at $5 \mu \mathrm{g} / \mathrm{ml}$, the final stimulation concentration, following which the UCMSCs were then seeded into a 6 -well plate at $1.5 \times 10^{5}$ concentration in $2 \mathrm{ml}$ of the medium.

Co-cultivation with peripheral blood leukocytes. Following the provision of informed consent, human peripheral blood leukocytes (PBLs) were acquired from two healthy donors (aged 25 and 26 years-old; male) and were labeled with carboxyfluorescein succinimidyl ester. The UCMSCs were co-cultured with the labeled PBLs (1:106) for $72 \mathrm{~h}$ at $37^{\circ} \mathrm{C}$, and cells were collected to detect the proliferation of PBLs using fluorescence-activated cell sorting (FACS; FC500 Cytomics; Beckman Coulter, Inc., Brea, CA, USA).

Flow cytometric analysis. To detect the surface markers of the MSCs, CD80, CD86, HLA-E, CD90, CD59 and CD29
(eBioscience, San Diego, CA, USA) were used. The UCMSCs treated with the TLR9 agonist and the control group were collected following $72 \mathrm{~h}$ stimulation at room temperature and incubated with specific antibodies for $30 \mathrm{~min}$ at room temperature. The cells were then analyzed using flow cytometry, gating cells according to fluorescence intensity, also performed in the control group.

$R T-q P C R$. Total RNA was extracted from confluent UCMSCs using TRIzol ${ }^{\circledR}$ reagent (Invitrogen Life Technologies, Carlsbad, CA, USA), and reverse-transcribed using a ReverTraAce qPCR RT kit (FSQ-101; Toyobo, Co. Ltd., Kagoshima, Japan) to synthesize cDNA under the following conditions: $65^{\circ} \mathrm{C}$ for $5 \mathrm{~min}, 37^{\circ} \mathrm{C}$ for $15 \mathrm{~min}$ and $98^{\circ} \mathrm{C}$ for $5 \mathrm{~min}$. RealMaster mix (SYBR Green; FP202; Tiangen Biotech, Co., Ltd., Beijing, China) was used to perform qPCR on $500 \mathrm{ng}$ cDNA under the following cycling conditions: $95^{\circ} \mathrm{C}$ for $30 \mathrm{sec}, 40$ cycles at $95^{\circ} \mathrm{C}$ for $30 \mathrm{sec}, 58^{\circ} \mathrm{C}$ for $30 \mathrm{sec}$ and $72^{\circ} \mathrm{C}$ for $30 \mathrm{sec}$, followed by a melt curve between 55 and $95^{\circ} \mathrm{C}$ in $0.5^{\circ} \mathrm{C}$ increments and $10 \mathrm{sec}$ intervals. Primers for IL-1 $\beta$, IL-6, IL-8, L-10, IL-12, IL-16, IFN- $\alpha$, IFN- $\beta$, IFN- $\gamma$, TGF- $\beta$, TNF- $\alpha$, iNOS, IP-10, MCP-1, MIP-1 $\alpha$, MIP-1 $\beta$, RANTES, MCP-3, P53, c-myc, cdc2, TCAM-1, Selectin-E, VCAM-1, CCL21, CCL24, CCL26 and GAPDH were used. The primer sequences were synthesized by Tsingke Biotech Company (Chengdu, China).

Antibody chip array. From the two groups of UCMSCs, the supernatant was collected following $4 \mathrm{~h}$ stimulation, according to the manufacturer's instructions of the RayBio Human Antibody Array C Series 1000 (RayBiotech Norcross, GA, USA), and the expression of proteins were analyzed.

Assessment of the effects of TLR activation on proliferation and migration of human UCMSCs. According to the manufacturer's instructions, a Cell-Light EdU Apollo 567 in vitro kit (RiboBio, Guangzhou, China) was used to assess UCMSC proliferation. The immunostained cells were visualized using a Bio-Rad A1S1 laser confocal microscope (Bio-Rad Laboratories, Inc.). UCMSC migration was examined using a Transwell assay/modified Boyden chamber. Briefly, 1x10 cells were seeded on the upper chamber in $1 \%$ serum-free Dulbecco's modified Eagle's medium (DMEM; Invitrogen Life Technologies), with or without the agonists. DMEM $(100 \mathrm{ml})$ supplemented with $10 \%$ fetal bovine serum (Invitrogen, Waltham, MA, USA) was added to the lower chamber. Subsequently, non-migratory cells were removed from the upper chamber, and the migratory cells were visualized using a Leica inverted microscope (DM4000B) after staining with crystal violet, and Leica Application Suite Advanced Fluorescence software (Leica Microsystems, Inc., Buffalo Grove, IL, USA).

Western blot analysis. The cells were harvested with $200 \mu \mathrm{l}$ radioimmunoprecipitation assay lysis buffer $(25 \mathrm{mM}$ Tris- $\mathrm{HCl}$ $\mathrm{pH}$ 7.6, $150 \mathrm{mM} \mathrm{NaCl}, 1 \% \mathrm{NP}-40,1 \%$ sodium deoxycholate, and $0.1 \%$ SDS), supplemented with a protease inhibitor (Sigma-Aldrich, St. Louis, MO, USA), following which the immunoreactive protein was detected using electrochemiluminescence on an X-ray film. Using ImageJ $1.48 \mathrm{u}$ software (National Institutes of Health, Bethesda, MD, USA), the 
resulting autoradiographs were scanned and quantified. The primary antibodies used for western blotting were as follows: Rabbit monoclonal CD29 (1:1,000; cat. no. 1798-1; Epitomics, Burlingame, CA, USA), rabbit monoclonal mitogen-activated protein kinase (MAPK)-p38 (1:1,000; cat. no. 1544-1; Epitomics) rabbit monoclonal $\beta$-catenin (1:5,000; cat. no. 1247-1; Epitomics) and mouse monoclonal GAPDH (1:2,000; cat. no. ab8245; Abcam).

Evaluation of UCMSC differentiation in vitro. To analyze UCMSC differentiation, conditioned media specific for chondrocytes (cat. no. A10071-01; Gibco-BRL), osteocytes (cat. no. A10072-011; Gibco-BRL) and adipocytes (cat. no. A10070-01; Gibco-BRL) were added to induce osteogenic and adipogenic differentiation of hUCMSCs. The two groups were treated with Oil Red O (Baso Diagnostics, Inc., Zhuhai, China) staining, Alizarin red staining (MeiLianShengWu, Shanghai, China) and safranine staining (Shanghai Kayon Biotechnology Co., Ltd., Shanghai, China) respectively after 3,10 and 14 days to detect adipogenesis, osteogenesis and chondrogenesis.

Statistical analysis. Data are expressed as the mean \pm standard error of the mean and were analyzed using SPSS 16.0 software (SPSS, Inc., Chicago, IL, USA). One-way analysis of variance was used for multiple comparisons, followed by a Dunnett's test to analyze the significance between two groups. $\mathrm{P}<0.05$ was considered to indicate a statistically significant difference.

\section{Results}

TLR9 ligand-regulated secretion of immunogenicity molecules. UCMSCs were stimulated by the TLR9-specific ligand $\mathrm{CpG}$ in order to detect changes in immune-associated molecule expression. From the UCMSCs treated with TLR9 agonist for varied durations (4, 12, 24, 72 and $120 \mathrm{~h})$, mRNA was isolated, which was assayed using RT-qPCR. The data indicated an increase following TLR9 exposure of several important expressed cytokines and chemokines, including mucosa-associated epithelial chemokine, thymus-expressed chemokine, Leuk, nuclear factor- $\kappa \mathrm{B}$, monocyte chemoattractant protein 2 , transforming growth factor (TGF- $\beta$ ) and other important immune factors (Fig. 1A). In addition, TLR9 stimulation markedly inhibited the expression of specific stem cell markers, and certain important tumor-associated factors were also induced by $\mathrm{CpG}$.

TLR9 ligand leads to activation of downstream signal molecules. To examine downstream signaling capabilities, the UCMSCs were stimulated between 4 and $120 \mathrm{~h}$ with TLR9 ligands and the results were assessed using western blot analysis. Several important kinase signal proteins, including $\beta$-catenin and CD29, as well as two members of the MAPK family, P38, were assessed. Treatment with the TLR9 agonist appeared to enhance the protein level of CD29, particularly at 24 and $72 \mathrm{~h}$ post-stimulation (Fig. 1A). Previous studies have confirmed that phosphorylated signal transducer and activator of transcription 3 , as the downstream signal pathway of interleukin (IL)-6 (16), MAPK-P38 and $\beta$-catenin decreases at different intervals. In MAPK detection in the present study, one important member, $\mathrm{p} 38$, exhibited no significant variation following TLR9 stimulation. This observation is significantly different from that of a previous study, which reported that the protein level of MAPK is promoted by TLR agonist treatment (17). The present study also found that the protein level of $\beta$-catenin first decreased at the 12- and 24-h intervals, but increased at $72 \mathrm{~h}$ (Fig. 1B). This suggested that treatment with the TLR9 agonist treatment did not affect these pathways.

Proliferation of PBL in co-culture with UCMSCs remains unchanged in the presence of TLR9 agonist. A number of ongoing clinical trials are based on the observation that MSCs can inhibit immune response (21). Therefore, the present study assessed whether this function of MSCs is compromised following addition of the TLR9 agonist. Initially, human PBLs were labelled with carboxyfluorescein succinimidyl ester (CFSE; Multiscience, Hangzhou, China) and co-cultured with UCMSCs, with or without TLR9 activation. The proliferation of PBLs was measured $72 \mathrm{~h}$ after co-culture, based on CFSE dilution. These experiments revealed that PBL proliferation did not vary significantly in the presence of the TLR9 agonist, compared with those without TLR9 ligands (Fig. 2A). The expression of co-stimulatory molecules in the UCMSCs exhibited almost no change in response to the TLR9 agonist (Fig. 2B). Therefore, the present results suggested that one of the mechanisms by which MSCs are not rejected or injured in vivo is their contact with TLR9 ligands. Antibody measurement confirmed the variation of secreted proteins (Fig. 2C), which were observed in Fig. 1.

TLR9 ligand CpG-ODN enables osteoblast differentiation of UCMSCs. To examine the effect of TLR9 on UCMSC differentiation, TLR9 agonist was added to the culture medium, as UCMSCs were induced by the conditioned medium to differentiate into osteocytes, chondrocytes and adipocytes. Osteocyte differentiation was detected, following supplementation of the cell culture media with osteogenic inducing medium via Alizarin red staining. Adipogenic differentiation, induced by adipogenic medium, appeared under Oil red $\mathrm{O}$ staining. UCMSCs grown in culture with chondrocyte-inducing media resulted in chondrogenic differentiation, which was detected by safranine. As shown in Fig. 3, the presence of the TLR9 agonist in the osteogenic medium markedly enhanced the level of calcium deposition, compared with conditioned medium without $\mathrm{CpG}$, particularly 10 and 14 days after differentiation. The control groups exhibited no marked differences in the presence of $\mathrm{CpG}$ in adipogenic and chondrogenic media (data not shown).

TLR9 activation inhibits hUCMSC proliferation and migration. The Cell-Light EdU Apollo 567 In Vitro kit and the transwell/modified Boyden chamber were used to investigate the proliferation and migration of UCMSCs in the present study. The data suggested that proliferation and migration were inhibited following stimulation by the TLR9 ligands (Fig. 4).

\section{Discussion}

MSCs have multiple functions and are considered to be important in prospective cell-based therapies. Understanding the 
A
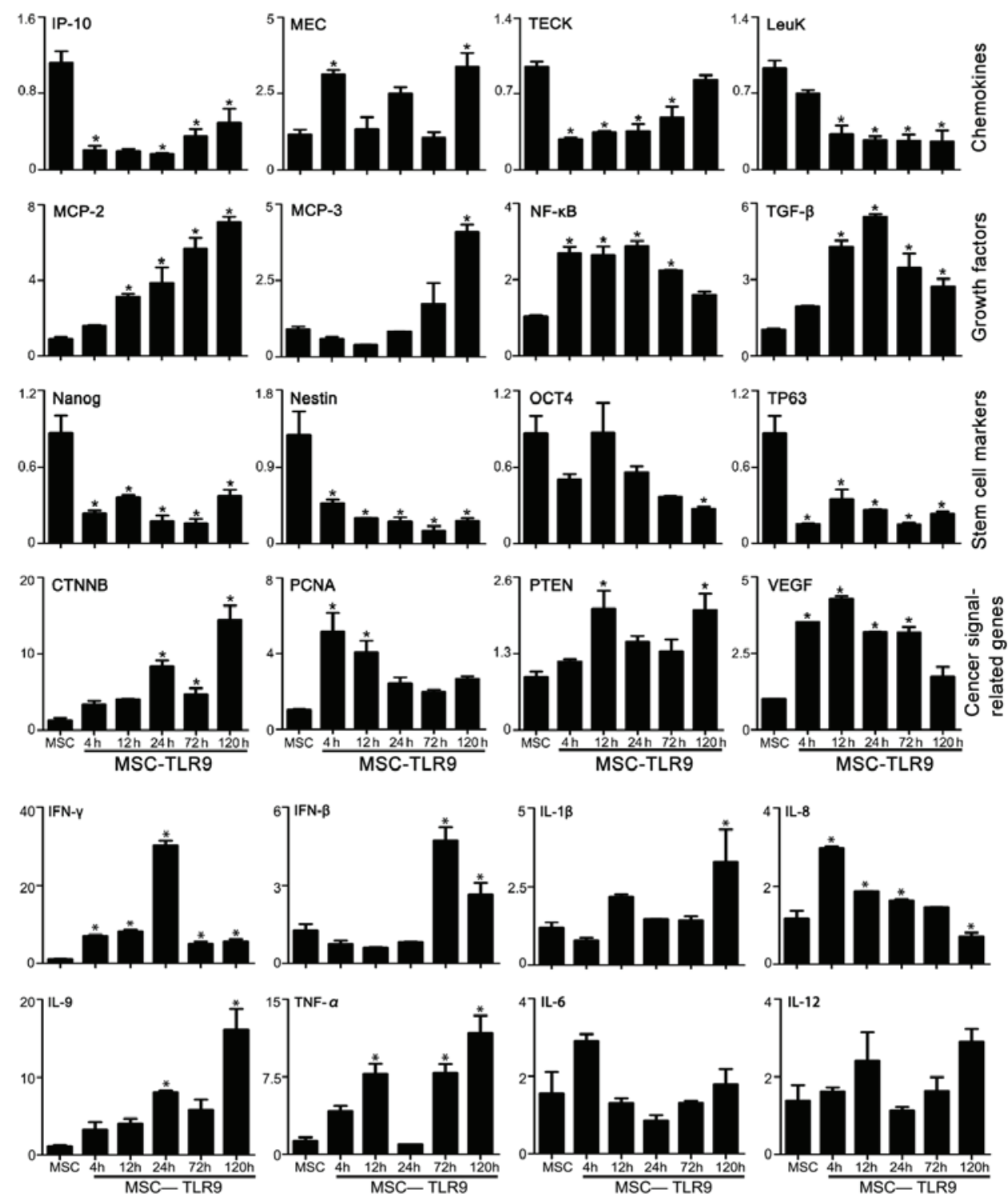

B

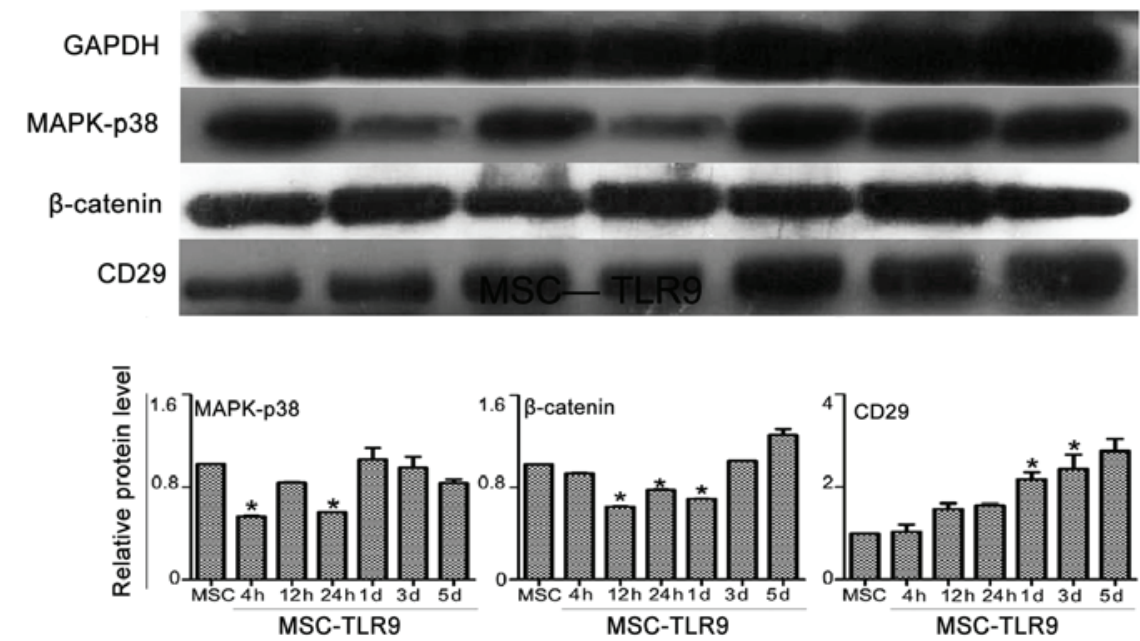

Figure 1. Detection of variations in gene expression and the expression of downstream signaling molecules and relative protein levels. (A) Gene expression levels were assessed using reverse transcription-quantitative polymerase chain reaction and (B) protein levels were detected using western blot analysis. The data are expressed as the mean \pm standard error of the mean. "P<0.05, vs. control. MSCs, mesenchymal stem cells; TLR9, Toll-like receptor 9; MAPK, mitogen-activated protein kinase; TGF, transforming growth factor; VEGF, vascular endothelial growth factor; NF, nuclear factor; MEC, mucosa-associated epithelial chemokine; TECK, thymus-expressed chemokine; MCP-2, monocyte chemoattractant protein 2; IFN, interferon; IL, interleukin; TNF, tumor necrosis factor. 
A

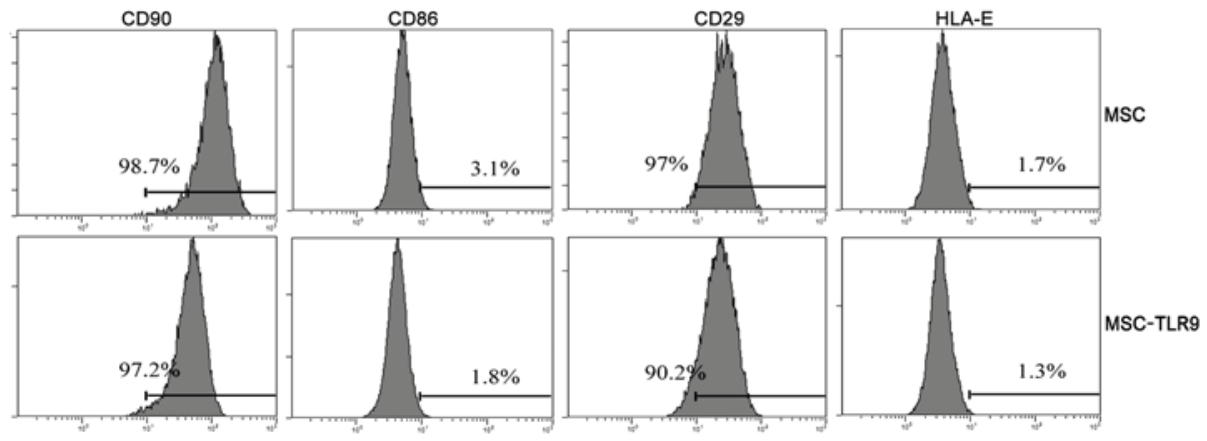

B
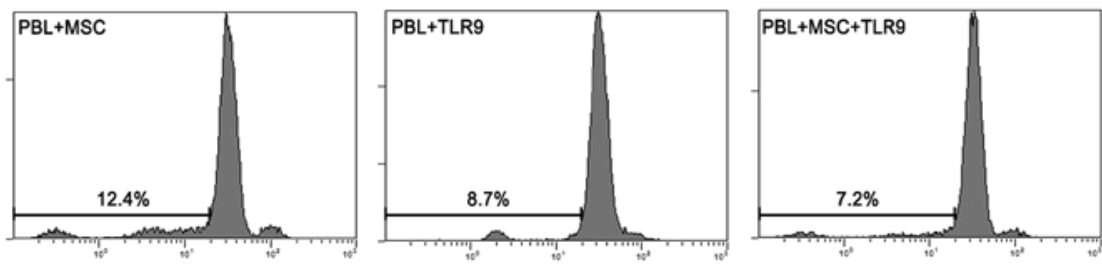

$\mathbf{C}$
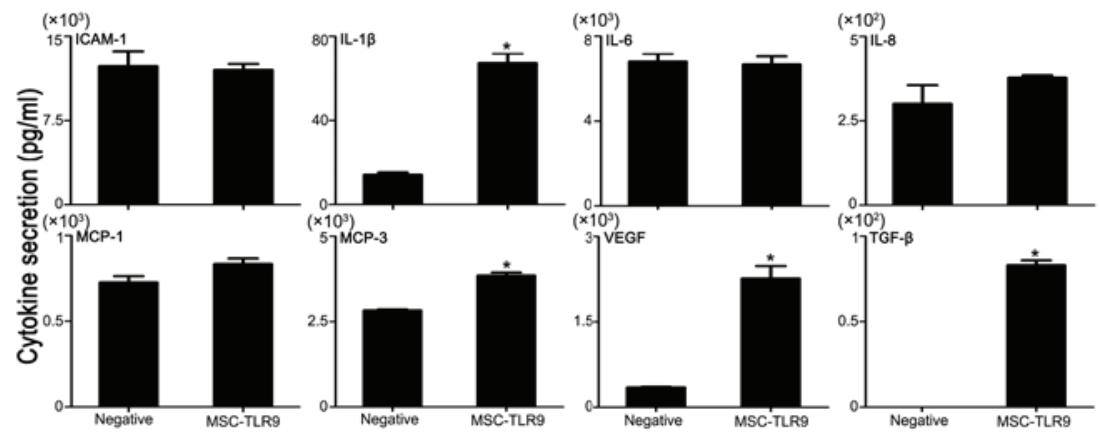

Figure 2. Co-culture of PBLs and MSCs. (A) Expression of co-stimulator and markers. PBL proliferation was not significantly altered in the presence of a TLR9 agonist. (B) Co-culture of PBLs and UCMSCs. The expression of co-stimulatory molecules exhibited almost no change in response to treatment with a TLR9 agonist. (C) Cytokine/chemokine secretion in culture medium. Data are expressed as the mean \pm standard error of the mean. "P<0.05, vs. negative group. UCMSCs, umbilical cord mesenchymal stem cells; PBL, peripheral blood leukocyte; MSCs, mesenchymal stem cells; Negative, untreated MSCs; TLR, Toll-like receptor.

factors and mechanisms, which regulate their ability to differentiate, self-renew, induce injury repair and suppress ongoing immune responses are crucial, and may allow the manipulation of MSCs for therapeutic use (22). However, several studies have suggested detrimental outcomes (23). Observations from human and animal studies have demonstrated the elimination of the majority of MSCs within a few days of infusion, indicating unknown host mechanisms of MSC recognition and rejection (24). Several other studies have also confirmed that MSCs alter immune status and cause immune responses, inducing the rejection and injury of MSCs (25-27).

TLRs are important in bridging innate and adaptive immune systems by recognizing conserved components from invasive pathogens (15). As UCMSCs have attracted increased attention in MSC-based therapy, the molecular details of the TLR agonist response are critical for improving controlled and desirable clinical outcomes. During cell therapy, engrafted MSCs may encounter molecular infectious agents, either in the blood circulation or in peripheral tissues, resulting in TLR activation. Numerous endogenous ligands of TLR, including heat shock protein (HSP)-60, HSP-70, heparin sulfate, fibronectin extra domain A, hyaluronan, oxidized LDL, uric acid, myeloid-associated proteins 8 and 14, intracellular components of fragmented cells, eosinophil-derived neurotoxin and human defensin-3 have been identified (28). These endogenous TLR ligands regulate the biological function of UCMSCs through endogenous stimuli during inflammation and tissue repair in the microenvironments of tissue injury and cell necrosis (29). An important aspect, which has not been previously investigated in detail is the activation and modulation of MSC activity by these danger signals.

Although a previous study indicated that TLRs have no effect on the immunogenicity of BMMSCs, the immunogenicity of TLRs in UCMSCs remains to be elucidated. The effect of the activation of TLR9 on the function of MSCs has been confirmed in a previous study (30). In the present study, functional TLR9 ligands were expressed in UCMSCs, and their activation by $\mathrm{CpG}-\mathrm{ODN}$ regulated $\mathrm{MSC}$ function, including cytokine release, multi-lineage differentiation, proliferation and migration.

MSCs are important in migration, expansion, apoptosis and immunomodulatory activities through the secretion of numerous growth factors, cytokines and chemokines (31). The present study demonstrated that stimulation of TLR9 ligands in UCMSCs elevated the secretion of pro-inflammatory cytokines (IL-1 $\beta$, IL- 8 , IL-9, interferon (IFN)- $\gamma$, IFN- $\beta$, TGF- $\beta$ and tumor necrosis factor- $\alpha$ ), excluding IL-6 and IL-12. Several important stem cell markers (Nanog, Nestin, OCT4 and TP63) were also markedly inhibited upon TLR9 stimulation. 

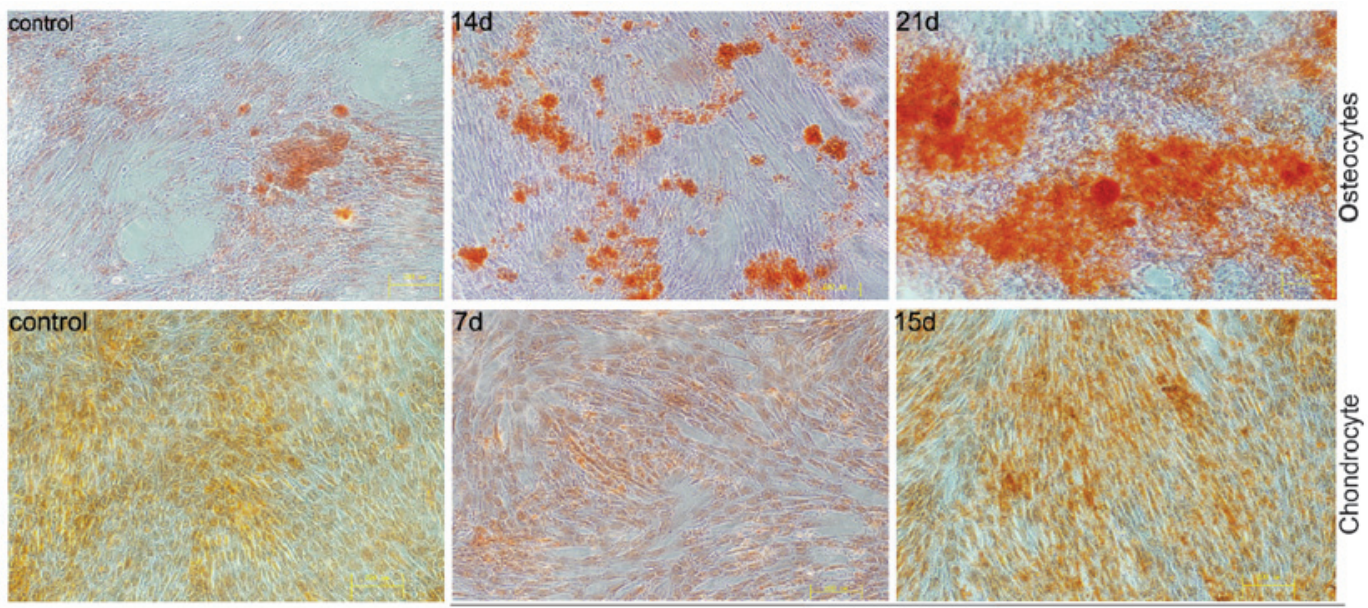

MSC-TLR9

Figure 3. Differentiation of umbilical cord mesenchymal stem cells in the presence of TLR9 agonist (Alizarine red and safranine staining; magnification, x200). MSCs, mesenchymal stem cells; TLR, Toll-like receptor; control, untreated MSCs; MSC-TLR, TLR9 agonist-treated MSCs.

A
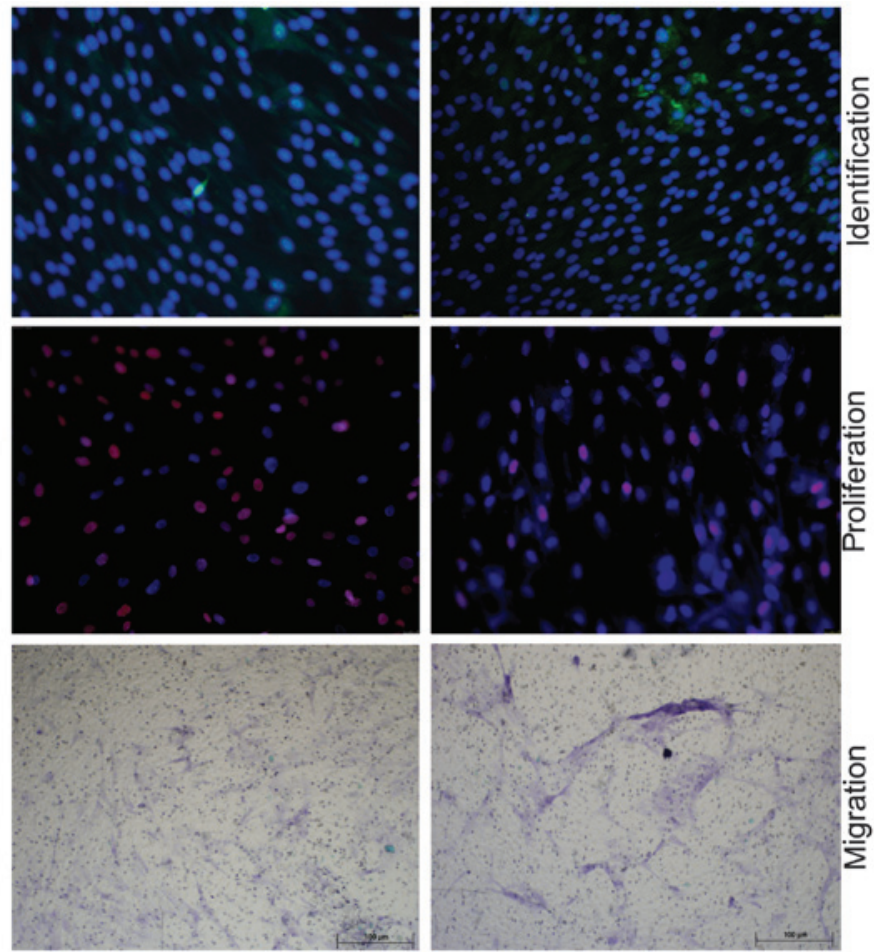

B
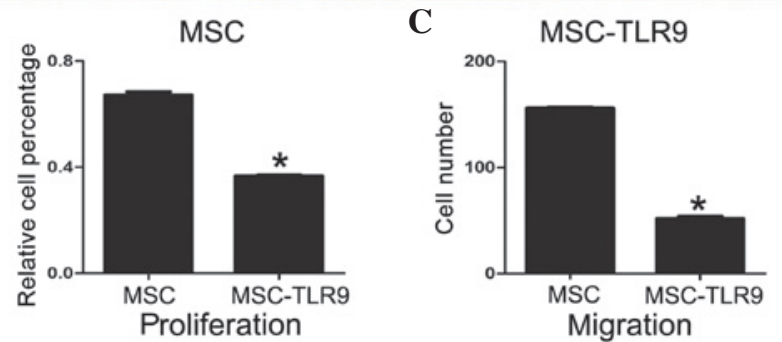

Figure 4. Variation detection using immunofluorescence. TLR9 activation inhibits UCMSC proliferation and migration. (A) Stage-specific embryonic antigen 4 was used to identify UCMSCs. (B) 5-ethynyl-2'-deoxyuridine was used for assessment the cell proliferation of UCMSCs. (C) Transwell assay was used for the analysis of migration. Data are expressed as the mean \pm standard error of the mean. ${ }^{*} \mathrm{P}<0.05$, vs. control group. UCMSCs, umbilical cord mesenchymal stem cells; MSCs, mesenchymal stem cells; TLR, Toll-like receptor; MSC-TLR9. TLR9 agonist-treated MSCs.

MSCs possess an increased tendency to enhance tumor sphere formation and tumor initiation (1). Interactions between MSCs and tumor cells involve MSC secretion of signaling molecules, including vascular endothelial growth factor (VEGF), IL-6, phosphatase and tensin homolog (PTEN) and CCLs, which stimulate various signaling pathways, particularly 
those associated with cell growth and apoptotic regulation in tumor cells (24). Similar to a previous study, the present study demonstrated that, in the presence of TLR9, detection of gene expression revealed upregulated secretion by tumor-initiation genes (PTEN, proliferating cell nuclear antigen, $\beta$-catenin and VEGF). The observation thatTLR9 activation-induced the expression of pro-inflammatory factors led to the hypothesis that exposure of UCMSCs to TLR9 agonists may increase the immunogenicity of UCMSCs. Therefore, co-cultures of PBLs and UCMSCs were performed in the present study to identify whether TLR9 increased the proliferation of PBLs. To measure the expression of co-stimulatory molecules, CD80, CD86 and HLA-II, FACS was also performed. The results revealed a negative effect of TLR9 on the immune status of UCMSCs. One basic characteristic of UCMSCs are their differentiation ability. As a previous study indicated, human AD-MSCs osteogenic differentiation increases in response to LPS and peptidoglycan activation, whereas CPG-ODN impairs differentiation (29). The results of the present study indicated that the presence of a TLR9 agonist enabled osteogenic differentiation.

The results of the present study demonstrated for the first time, to the best of our knowledge, TLR9 regulation of immune status and biological function. Several paths of evidence were pursued. Firstly, TLR9 was observed to increase the expression of pro-inflammatory molecules and decrease the expression of stem cell markers. Subsequently, the marked effects of TLR9 agonist treatment on UCMSCs were observed to inhibit proliferation and migration. Finally, the differentiation results confirmed that the TLR9 agonist increased osteoblast differentiation capability. In conclusion, the present study provided a novel observation of TLR9 mediating immune modulator response via UCMSCs, critical to MSC-based therapy design. As UCMSCs are attracting more attention as a replacement for BMMSCs, another important aspect of the present study is that MSCs isolated from umbilical tissue were used. Understanding how factors and mechanisms regulating biological functions, including differentiation, self-renewal and involvement in immune responses may allow the manipulation of UCMSCs for clinical use.

The present study critically implicated TLR9 as a vital modulator in UCMSC function, including differentiation, proliferation and migration. Further characterization of TLR9-activated UCMSCs using in vivo experimental systems is required to establish the physiological role of TLRs in the regulation of UCSMC function.

\section{Acknowledgements}

This study was supported by the National Natural Scientific Foundations of China (grant no. 81200315) and the China Postdoctoral Science Foundation Grants (grant nos. 2011M501413 and 2013T60855).

\section{References}

1. Phinney DG and Prockop DJ: Concise review: mesenchymal stem/multipotent stromal cells: the state of transdifferentiation and modes of tissue repair - current views. Stem Cells 25: 2896-2902, 2007.
2. Pittenger MF, Mackay AM, Beck SC, Jaiswal RK, Douglas R, Mosca JD, Moorman MA, Simonetti DW, Craig S and Marshak DR: Multilineage potential of adult human mesenchymal stem cells. Science 284: 143-147, 1999.

3. Han KH, Ro H, Hong JH, Lee EM, Cho B, Yeom HJ, Kim MG, $\mathrm{Oh} \mathrm{KH}$, Ahn $\mathrm{C}$ and Yang J: Immunosuppressive mechanisms of embryonic stem cells and mesenchymal stem cells in alloimmune response. Transpl Immunol 25: 7-15, 2011.

4. Nagaya N, Fujii T, Iwase T, Ohgushi H, Itoh T, Uematsu M, Yamagishi M, Mori H, Kangawa K and Kitamura S: Intravenous administration of mesenchymal stem cells improves cardiac function in rats with acute myocardial infarction through angiogenesis and myogenesis. Am J Physiol Heart Circ Physiol 287: H2670-H2676, 2004.

5. Tatebe M, Nakamura R, Kagami H, Okada K and Ueda M: Differentiation of transplanted mesenchymal stem cells in a large osteochondral defect in rabbit. Cytotherapy 7: 520-530, 2005.

6. Im GI, Kim DY, Shin JH, Hyun CW and Cho WH: Repair of cartilage defect in the rabbit with cultured mesenchymal stem cells from bone marrow. J Bone Joint Surg Br 83: 289-294, 2001.

7. Bensaïd W, Oudina K, Viateau V, Potier E, Bousson V, Blanchat C, Sedel L, Guillemin G and Petite H: De novo reconstruction of functional bone by tissue engineering in the metatarsal sheep model. Tissue Eng 11: 814-824, 2005.

8. Harris CT and Cooper LF: Comparison of bone graft matrices for human mesenchymal stem cell-directed osteogenesis. J Biomed Mater Res A 68: 747-755, 2004.

9. Horwitz EM, Gordon PL, Koo WK, Marx JC, Neel MD, McNall RY, Muul L and Hofmann T: Isolated allogeneic bone marrow-derived mesenchymal cells engraft and stimulate growth in children with osteogenesis imperfecta: Implications for cell therapy of bone. Proc Natl Acad Sci USA 99: 8932-8937, 2002.

10. Kawada H, Fujita J, Kinjo K, Matsuzaki Y, Tsuma M, Miyatake H, Muguruma Y, Tsuboi K, Itabashi Y, Ikeda Y, et al: Nonhematopoietic mesenchymal stem cells can be mobilized and differentiate into cardiomyocytes after myocardial infarction. Blood 104: 3581-3587, 2004

11. Van Damme A, Vanden Driessche T, Collen D and Chuah MK: Bone marrow stromal cells as targets for gene therapy. Curr Gene Ther 2: 195-209, 2002

12. Allison M: Genzyme back Osiris, despite Prochymal flop. Nat Biotechnol 27: 966-967, 2009.

13. Huang XP, Sun Z, Miyagi Y, McDonald Kinkaid H, Zhang L, Weisel RD and Li RK: Differentiation of allogeneic mesenchymal stem cells induces immunogenicity and limits their long-term benefits for myocardial repair. Circulation 122: 2419-2429, 2010

14. Blasius AL and Beutler B: Intracellular toll-like receptors. Immunity 32: 305-315, 2010.

15. Akira S, Uematsu S and Takeuchi O: Pathogen recognition and innate immunity. Cell 124: 783-801, 2006.

16. Lombardo E, DelaRosa O, Mancheño-Corvo P, Menta R, Ramírez C and Büscher D: Toll-like receptor-mediated signaling in human adipose-derived stem cells: Implications for immunogenicity and immunosuppressive potential. Tissue Eng Part A 15: 1579-1589, 2009.

17. Cassatella MA, Mosna F, Micheletti A, Lisi V, Tamassia N, Cont C, Calzetti F, Pelletier M, Pizzolo G and Krampera M: Toll-like receptor-3-activated human mesenchymal stromal cells significantly prolong the survival and function of neutrophils. Stem Cells 29: 1001-1011, 2011.

18. Chen W, Liu J, Manuchehrabadi N, Weir MD, Zhu Z and $\mathrm{Xu} \mathrm{HH}$ : Umbilical cord and bone marrow mesenchymal stem cell seeding on macroporous calcium phosphate for bone regeneration in rat cranial defects. Biomaterials 34: 9917-9925, 2013.

19. Fong CY, Chak LL, Biswas A, Tan JH, Gauthaman K, Chan WK and Bongso A: Human Wharton's jelly stem cells have unique transcriptome profiles compared to human embryonic stem cells and other mesenchymal stem cells. Stem Cell Rev 7: 1-16, 2011.

20. Le Blanc K, Frassoni F, Ball L, Locatelli F, Roelofs H, Lewis I, Lanino E, Sundberg B, Bernardo ME, Remberger M, et al; Developmental Committee of the European Group for Blood and Marrow Transplantation: Mesenchymal stem cells for treatment of steroid-resistant, severe, acute graft-versus-host disease: A phase II study. Lancet 371: 1579-1586.

21. Shi M, Liu ZW and Wang FS: Immunomodulatory properties and therapeutic application of mesenchymal stem cells. Clin Exp Immunol 164: 1-8, 2011. 
22. Pevsner-Fischer M, Morad V, Cohen-Sfady M, Rousso-Noori L, Zanin-Zhorov A, Cohen S, Cohen IR and Zipori D: Toll-like receptors and their ligands control mesenchymal stem cell functions. Blood 109: 1422-1432, 2007.

23. Shi Y, Su J, Roberts AI, Shou P, Rabson AB and Ren G: How mesenchymal stem cells interact with tissue immune responses. Trends Immunol 33: 136-143, 2012.

24. Kaplan JM, Youd ME and Lodie TA: Immunomodulatory activity of mesenchymal stem cells. Curr Stem Cell Res Ther 6: 297-316, 2011

25. Spaggiari GM, Capobianco A, Becchetti S, Mingari MC and Moretta L: Mesenchymal stem cell-natural killer cell interactions: Evidence that activated NK cells are capable of killing MSCs, whereas MSCs can inhibit IL-2-induced NK-cell proliferation. Blood 107: 1484-1490, 2006.

26. Li Y and Lin F: Mesenchymal stem cells are injured by complement after their contact with serum. Blood 120: 3436-3443, 2012.
27. Nauta AJ, Westerhuis G, Kruisselbrink AB, Lurvink EG, Willemze R and Fibbe WE: Donor-derived mesenchymal stem cells are immunogenic in an allogeneic host and stimulate donor graft rejection in a nonmyeloablative setting. Blood 108: 2114-2120, 2006

28. Kawai T and Akira S: The role of pattern-recognition receptors in innate immunity: Update on Toll-like receptors. Nat Immunol 11: 373-384, 2010

29. Torsvik A and Bjerkvig R: Mesenchymal stem cell signaling in cancer progression. Cancer Treat Rev 39: 180-188, 2013.

30. DelaRosa O and Lombardo E: Modulation of adult mesenchymal stem cells activity by toll-like receptors: Implications on therapeutic potential. Mediators Inflamm 2010: 865601, 2010.

31. Hsieh JY, Wang HW, Chang SJ, Liao KH, Lee IH, Lin WS, Wu CH, Lin WY and Cheng SM: Mesenchymal stem cells from human umbilical cord express preferentially secreted factors related to neuroprotection, neurogenesis, and angiogenesis. PLoS One 8: e72604, 2013. 